\title{
Evaluation of optical inspection methods for non-destructive assessment of embedded microstructures and defects in ceramic materials
}

\author{
R. Su, L. Mattsson \\ Industrial Metrology and Optics, Production Engineering, KTH Royal Institute of Technology, S-100 44 Stockholm, \\ Sweden
}

\begin{abstract}
The future ceramic micro processing based on tape stacking requires the development of inspection systems to perform high-resolution in-process quality control of embedded manufactured cavities, metal structures and defects. This paper presents non-destructive techniques for monitoring processes and controlling the different steering parameters. Results are shown for optical coherence tomography (OCT), IR-transmission and reflection measurement, and X-ray micro computed tomography. Suitable working conditions are analyzed to improve the detection performance.
\end{abstract}

Keywords: Ceramic processing, Non-destructive testing (NDT), Optical inspection, Micrometrology

\section{Introduction}

The "roll-to-roll" 3D shaping technology is a promising solution for large-scale and cost-effective manufacture of ceramic micro devices. One development activity in this area is the EU project Multilayer [1], which also addresses the high demands on quality inspection and process control.

Obviously optical inspection methods have great advantages as non-destructive high-speed and high-resolution measurement tools For instance, tape thickness can be measured using dual confocal chromatic probe with operating distances in the $\mathrm{mm}$ range and vertical resolution of the order of nanometers depending on maximum measurement range. Lateral resolution can be of the order of micrometers. Confocal microscopy and chromatic white light sensor can be used for measuring the dimensions of the micro structures in the free surface with measuring rate of up to $14 \mathrm{kHz}$. Lateral dimensions of processed micro features can be measured by optical microscopy using telecentric lenses which may reduce perspective error and distortion, increase image resolution, and keep constant magnification independent of shift in image and/or object planes. Moreover, camera inspection or laser scanner is suggested for measuring length, width, height, diameter, squareness, flatness and 3D shape, as well as position and orientation.

In this paper we focus on optical coherence tomography (OCT) and IR-transmission and reflection measurement which are promising techniques for in-process monitoring but have not been commercially available in ceramic industry. Xray computed microtomography is also evaluated due to its high resolution and extraordinary penetration capability in ceramics.

\section{Suitable working conditions}

Different optical inspection techniques rely on the different characteristics of light/radiation-sample interactions that include reflection and transmission, and light scattering on the surface or inside the materials. Also the wavelength $(\lambda)$ of the light source of the specific optical inspection technique is a crucial parameter.

\subsection{Optical coherence tomography (OCT)}

Owing to the development of low-coherence light source and fast scanning mechanics OCT based on low coherence interferometry provides high resolution and high speed $3 \mathrm{D}$ imaging, and has been actively used in the biomedical area [2].

In OCT detection the photons carrying the spatial location information of internal structures have to fulfil the criterion of single and least scattered photons. For both alumina and zirconia (highly scattering materials in the visible) the absorption can be neglected in the wavelength region $\lambda=0.5$ to $5 \mu \mathrm{m}$. The scattering properties are the most important factor determining the probing depth which is one of the bottlenecks of OCT imaging. As shown in Fig. 1, for both alumina and zirconia the scattering coefficient decreases with increasing wavelength. Without changing the other conditions, smaller scattering coefficient can benefit the OCT imaging contrast and enhance the probing depth, i.e. longer wavelength should be chosen. The centre operating wavelengths of existing OCT systems suitable for ceramics are presented also in this figure. It is worth to mention that a laboratory OCT system working at $6 \sim 8 \mu \mathrm{m}$ has been developed recently [3], and the light extinction coefficient in this wavelength region (not plotted here) is around $0.18 \mathrm{~mm}^{-1}$ for alumina considering both scattering and absorption. These operating wavelengths may significantly contribute to the performance of OCT.

\subsection{IR-transmission measurement}

The total and diffuse transmittance and reflectance can be measured by a spectrophotometer with an integrating sphere in visible and infrared region. [4]

As shown in Fig 1 the scattering decreases at longer wavelengths. This enhances the collimated in-line (direct) transmission up to where absorption sets in. However, to get a high sensitivity of thickness determination vs in-line transmission measurements we need to be at an intermediate scattering or absorption level. 


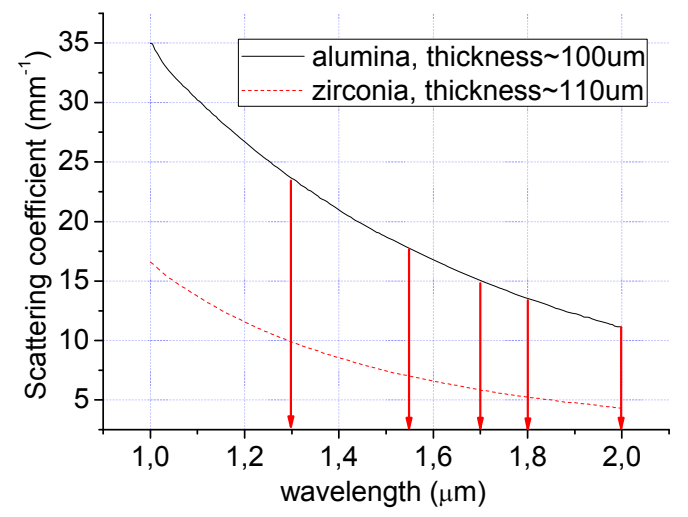

Fig. 1 Scattering coefficient versus wavelength. The existing OCT systems suitable for ceramics operate at $1.3,1.55,1.7,1.8$, and $2 \mu \mathrm{m}$.

By measuring in-line transmission of two samples with the same scattering properties but different thickness we could derive a thickness transmittance "contrast" function vs. wavelength. This is shown in Fig. 2. For sufficiently long wavelengths the in-line transmittance contrast is calculated as,

$$
\mathrm{C}=\left(\mathrm{T}_{\max }-\mathrm{T}_{\min }\right) \times 100 \%
$$

Where $T_{\max }$ and $T_{\min }$ is the in-line transmittance of a ceramic sample with thickness around $200 \mu \mathrm{m}$ and $350 \mu \mathrm{m}$, respectively.

The contrast peaks shows that $\lambda \sim 3.4 \mu \mathrm{m}$ is most suitable for alumina, and $\lambda \sim 2 \mu \mathrm{m}$ for zirconia. Although the contrasts also have peaks at longer wavelengths caused by absorption, the interference effects seen in the transmission signal of the zirconia may reduce the measurement accuracy.

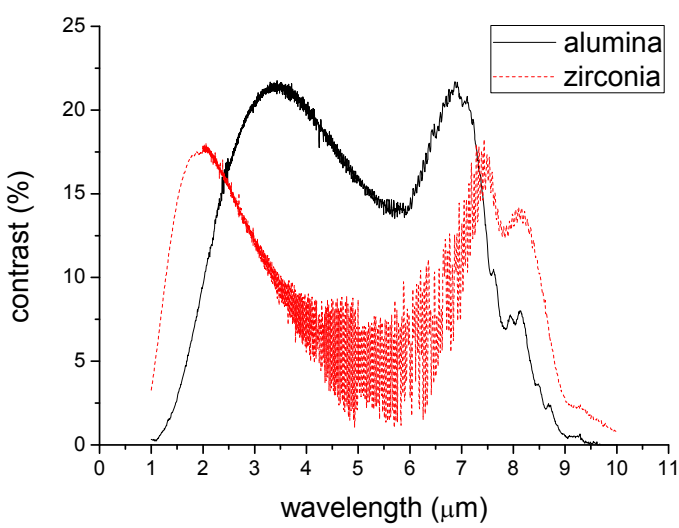

Fig. 2 Wavelength dependence of in-line transmittance "contrast" for two different thicknesses for sintered tapes of alumina and zirconia in IR region.

\section{Results}

\subsection{IR Optical Coherence Tomography}

Among the existing commercial and research lab OCT systems, the scanning rate for a single Ascan can vary from $8 \mathrm{kHz}$ to over $300 \mathrm{kHz}$ [2]. The typical axial and lateral resolutions are $12 \mu \mathrm{m}$ (in air) and $15 \mu \mathrm{m}$, respectively, and the typical maximum scanning depth is $3 \mathrm{~mm}$. According to our experimental results, the maximum detectable thickness at $\lambda=1.3 \mu \mathrm{m}$ of alumina tapes sintered at $1630{ }^{\circ} \mathrm{C}$ is around $260 \mu \mathrm{m}$, and $500 \mu \mathrm{m}$ for sintered zirconia tapes.

The thickness can be calculated by dividing the measured optical path length between two boundaries by the refractive index of the material. Dimensions of the structured channel embedded in highly-scattering alumina ceramic material can also be measured in cross sectional OCT images. [5]

As shown in Fig. 3, the measured thickness of the top layer and the depth of the channel are $102 \mu \mathrm{m}$ and $79 \mu \mathrm{m}$, respectively. The width of the channel is around $170 \mu \mathrm{m}$ at the top and $71 \mu \mathrm{m}$ at the bottom.

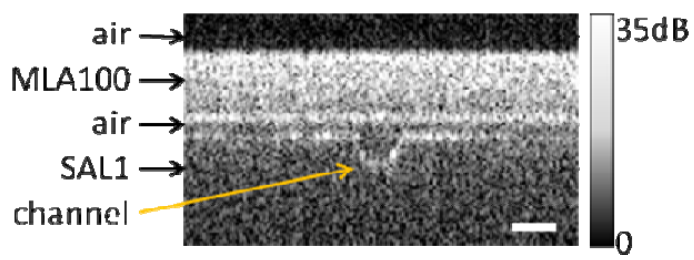

Fig. 3 the cross-sectional OCT image of an embedded laser-machined channel. The scale bar is $150 \mu \mathrm{m}$ for both axial (optical distance is considered) and lateral direction. (Thorlabs OCT was used)

As shown in Fig. 4, the front and rear surfaces can be clearly observed and the sample is tilted to reduce the specular reflected component. Two extraordinary bright spots can be found between the first and second surface. This is probably due to the strong backscattering at the interface between alumina and large air bubble with diameter of $15 \sim 20 \mu \mathrm{m}$, where the difference of refractive indices is large.

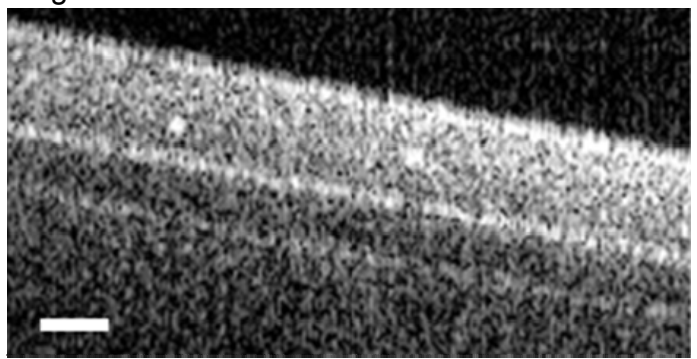

Fig. 4 Cross-sectional OCT image of a sintered alumina layer MLA100. The scale bar is $150 \mu \mathrm{m}$ for both axial (optical distance is considered) and lateral direction. (Thorlabs OCT was used)

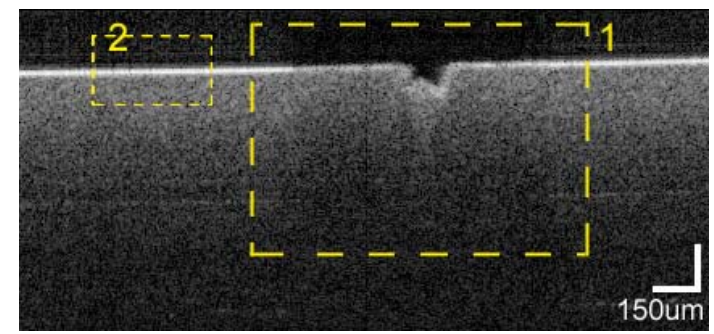

Fig. 5 Cross-sectional OCT image of a lasermachined alumina sample. Note the brightness change in the surface for area 1 and 2 . The vertical bar presents the optical distance. (Michelson Diagnostics OCT was used) 
During laser-machining of alumina green tape preheating and vaporization can cause material to build up and add roughness to areas close to the processed area. We have investigated this effect on areas close to laser machined channels and showed that the rough surface can be detected as a reduced OCT signal (Fig. 5). [6]

\subsection{IR transmission and reflection measurement}

As discussed in 2.2 in-line transmittance is a function of the thickness of ceramic tape. Thus, the tape thickness can be monitored by the measured in-line transmittance. In Fig. 6 the relations between the in-line transmittances and thicknesses are plotted for alumina and zirconia respectively at $\lambda=3 \mu \mathrm{m}$ and $1.8 \mu \mathrm{m}$. If the material is changed, this curve has to be calibrated again.

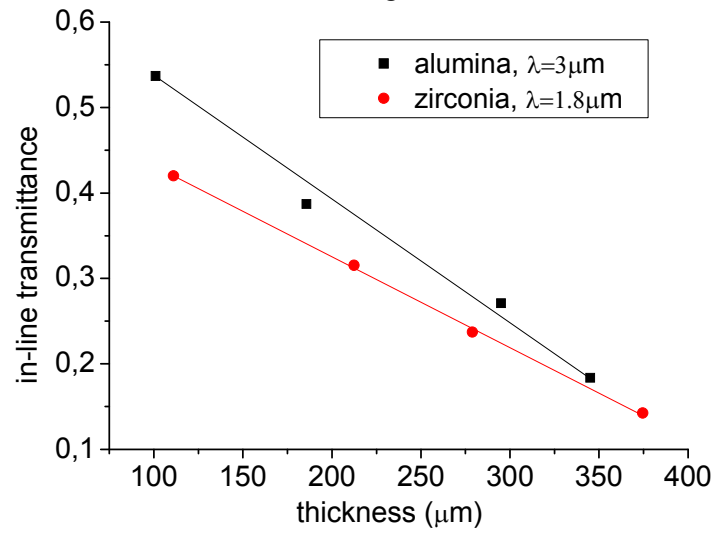

Fig. 6 The relation between measured in-line transmittance and the thickness of sintered alumina and zirconia.

IR-transmission obtained with an IRthermovision camera can be used for detecting metal contaminations or embedded metal structures in a stack of ceramic tapes.

In the preliminary tests at Innventia $A B$, we used a FLIR camera with a cooled InSb detector sensitive to $\lambda 3-5 \mu \mathrm{m}$. With a frame rate of $15 \mathrm{~Hz}$ and a frame size of $640 \times 512$ pixels it can be used for screening of the roll-to-roll tape process.

Fig. 7 shows its capability of detecting silver prints (the line width is around $290 \mu \mathrm{m}$ ) on the surface (a) of an alumina plate or an embedded silver print in the multilayered ceramic stack (b). The embedded silver line can be detected without obvious distortion caused by the scattering.

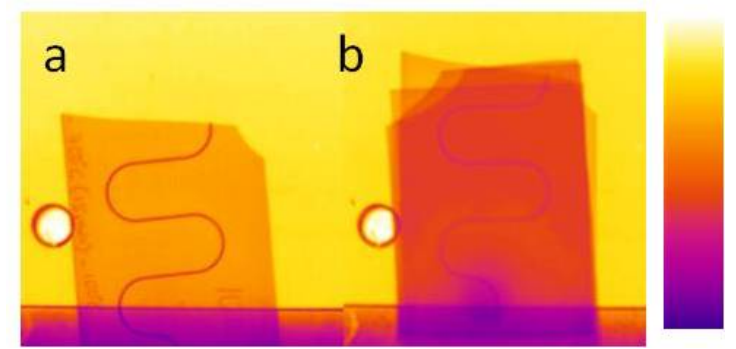

Fig. 7 A sintered alumina plate (a) with a silver printed line on top, b) with 3 additional sintered ceramic layers on top of the silver line, corresponding to an embedded depth of $800 \mu \mathrm{m}$. Image courtesy of Caroline Hyll, Innventia AB.
A rapid and simple method to assess the rootmean-square (rms) surface roughness of a sample is to the total integrated scattering (TIS) [7]. However, sintered alumina is translucent so that a large fraction of the total reflection comes from the bulk scattering, and the technique can therefore not be directly applied. However with a well determined refractive index of alumina or zirconia we know the specular Fresnel reflectance from the top surface. By measuring the loss of specular reflectance (i.e. TIS) relative the Fresnel reflectance we can get a value of the surface roughness. The loss is obtained by measuring the specular reflectance as the difference between total and diffuse reflectance using a spectrophotometer provided with an integrating sphere.

The measured TIS in specular direction of the sintered alumina tape with $200 \mu \mathrm{m}$ thickness is shown in Fig. 8 (dots). According to the scalar scattering theory the solid line calculated from rms roughness of $70 \mathrm{~nm}$ fits the measured scattering curve very well. This value agrees with the result measured by the KTH-Talystep surface profilometer. The deviation in the near infrared region is probably caused by dust particles on the sample surface and produce extra scattering.

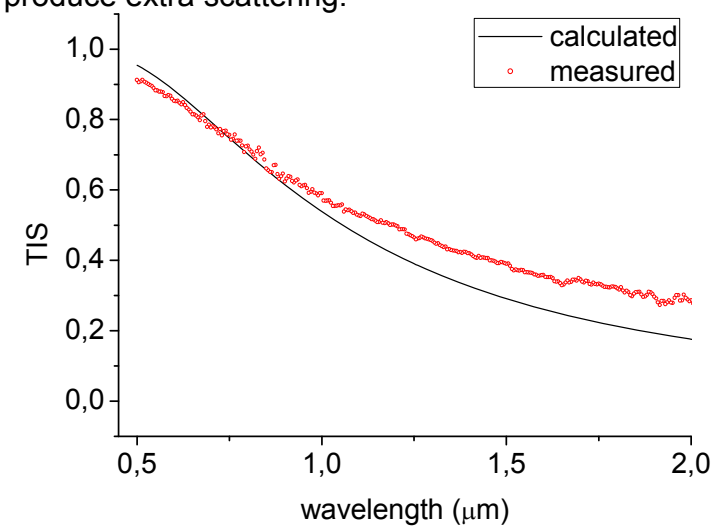

Fig. 8 Scattering loss (TIS) in specular direction of alumina sample versus wavelength. The rms roughness is obtained by fitting with calculated curve.

\subsection{X-ray micro CT}

X-ray micro computed tomography can provide non-destructive 3D porosity, phase and microstructure characterization of ceramics, composites, and polymers. The technique overcomes the limitation of traditional destructive mechanical slicing or chemical etching to obtain serial sectioning followed by imaging with SEM or optical microscopy. [8]

Tests were performed for sintered and green alumina tapes with MicroXCT-200 (Xradia) at Innventia $A B$. The transverse images of the sintered alumina tapes with embedded silver print and lasercut channel are shown in Fig. 9. The quality of the printed silver line and the rough surface of the channel bottom are directly observed.

The resolution of these tests is set to be around $5 \mu \mathrm{m}$. To achieve $1 \mu \mathrm{m}$ resolution of the Micro X-ray $\mathrm{CT}$, the samples must be prepared carefully with a dimension around $1 \mathrm{~mm}$ in the projection direction. A disadvantage is that the data acquisition time is very 
long, at present several hours (depends on the selected resolution). That is by far too long to be realistic for in-process metrology, but it can be utilized for off-line material characterization when problems related to internal features need to be solved. A colour change of the ceramic tape was observed after the intense X-ray exposure.

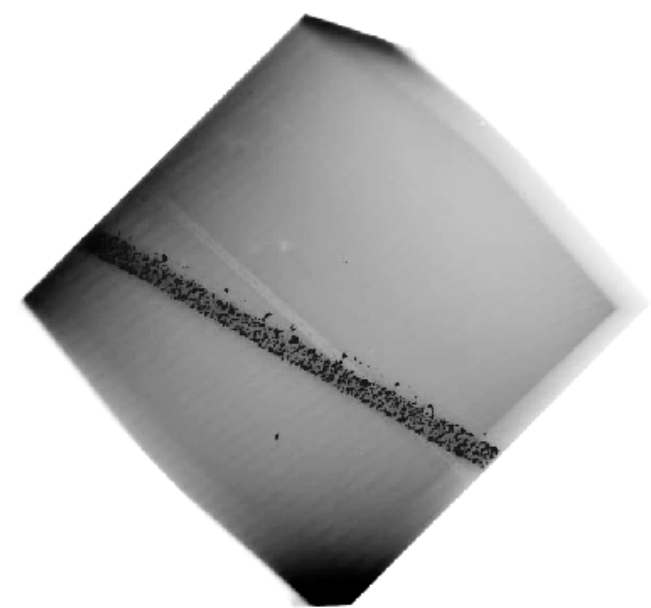

(a)

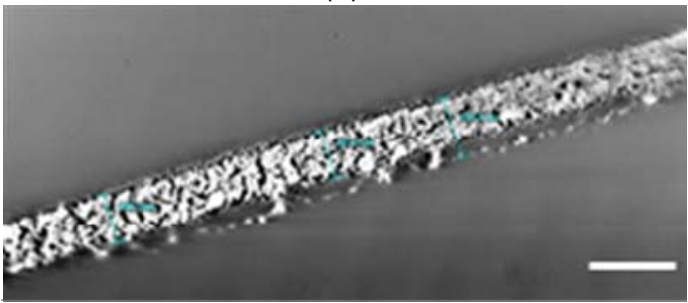

(b)

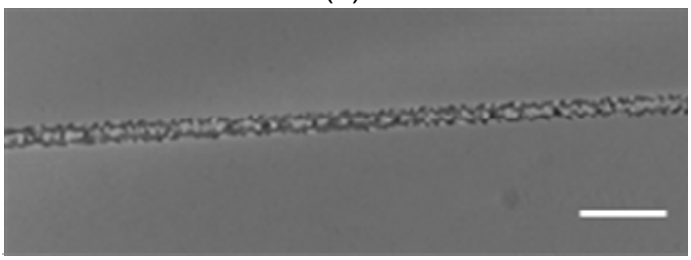

(c)

Fig. 9 The 3D (a) and transverse x-ray micro tomography images of three-layer sintered alumina stack with embedded silver print (b) and laser-cut channel (c). Scale bars are $500 \mu \mathrm{m}$. Image courtesy of Joanna Hornatowska, Innventia AB.

\section{Discussion and conclusion}

Based on the optical properties of the alumina and zirconia ceramic materials, the suitable working wavelength for OCT imaging and IR transmission detection has been analyzed. We demonstrate the experimental results in order to promote these techniques for the future demands in in-process, non-contact and high-resolution 3D monitoring of embedded ceramic micro structures. X-ray micro CT has also been evaluated as an off-line measurement method providing high resolution and excellent penetration.

Particularly for the OCT technique, there is no commercial setup operating at the most suitable wavelength because OCT was originally developed for using in biomedicine area. However, according to the analysis in this paper it will be interesting to extend its working wavelength to mid-infrared region in order to achieve a larger probing depth in ceramics.

\section{References}

1. S. Bredeau and L. Federzoni, "Multilayer: a large scale production of micro devices via new rolled multi material layered 3D shaping technology," in Proceedings of the 4M/ICOMM 2009 Conference, (Karlsruhe, 2009), 419-422.

2. B. E. Bouma and G. J. Tearney, Handbook of optical coherence tomography, (Marcel Dekker, 2002).

3. C. S. Colley, J. C. Hebden, and D. T. Delpy, "Midinfrared optical coherence tomography," Rev. Sci. Instrum. 78, 123108 (2007).

4. A. Roos, "Use of an integrating sphere in solar energy research," Sol. Energy Mater. Sol. Cells 30, 77-94 (1993).

5. Rong Su, Mikhail Kirillin, Peter Ekberg, Arne Roos, Ekaterina Sergeeva, and Lars Mattsson, "Optical coherence tomography for quality assessment of embedded microchannels in alumina ceramic," Opt. Express 20, 4603-4618 (2012)

6. R. Su, M. Kirillin, D. Jurków, K. Malecha, L. Golonka, and L. Mattsson, "Optical coherence tomography - a potential tool for roughness assessment of free and embedded surfaces of laser-machined alumina ceramic," in Proceedings of the 4M 2011 Conference.

7. J.M. Bennett, and L. Mattsson, Introduction to surface roughness and scattering $2^{\text {nd }}$ ed., (OSA, 1999).

8. S. R. Stock, Micro Computed Tomography: Methodology and Applications, (CRC Press, 2008).

\section{Acknowledgements}

The authors wish to thank Swerea IVF AB for providing the ceramic samples, Thorlabs and Michelson Diagnostics Ltd for the OCT images, prof. Arne Roos, Uppsala University for the spectrophotometry measurement, and Caroline Hyll and Johanna Hornatowska at Innventia $A B$ for the IRimages and x-ray microtomography.

This work is supported by the Multilayer project (FP7-NMP4-2007-214122). 